\title{
Verification of Dosimetric and Positional Accuracy of Dynamic Tumor Tracking Intensity Modulated Radiation Therapy
}

\author{
Masaki Sueoka ${ }^{1,2 *}$, Akira Sawada ${ }^{3}$, Hiroaki Tanabe' ${ }^{2}, Y_{k i}$ Okada $^{2}$, Sho Taniuchi², \\ Noboru Okuuchi' ${ }^{2}$, Masao Tanooka ${ }^{1}$, Masaki Kokubo ${ }^{4}$, Koichiro Yamakado ${ }^{1}$ \\ ${ }^{1}$ Department of Radiology, Hyogo College of Medicine, Nishinomiya, Japan \\ ${ }^{2}$ Department of Radiological Technology, Kobe City Medical Center General Hospital, Kobe, Japan \\ ${ }^{3}$ Department of Radiological Technology, Faculty of Medical Science, Kyoto College of Medical Science, Nantan, Japan \\ ${ }^{4}$ Department of Radiation Oncology, Kobe City Medical Center General Hospital, Kobe, Japan \\ Email: *masaki.sueoka@gmail.com
}

How to cite this paper: Sueoka, M., Sawada, A., Tanabe, H., Okada, Y., Taniuchi, S., Okuuchi, N., Tanooka, M., Kokubo, M. and Yamakado, K. (2019) Verification of Dosimetric and Positional Accuracy of Dynamic Tumor Tracking Intensity Modulated Radiation Therapy. International Journal of Medical Physics, Clinical Engineering and Radiation Oncology, 8, 211-224.

https://doi.org/10.4236/ijmpcero.2019.84019

Received: September 4, 2019

Accepted: October 8, 2019

Published: October 11, 2019

Copyright ( 2019 by author(s) and Scientific Research Publishing Inc. This work is licensed under the Creative Commons Attribution International License (CC BY 4.0).

http://creativecommons.org/licenses/by/4.0/

(c) (i) Open Access

\begin{abstract}
Purpose: We performed both, dosimetric and positional accuracy verification of dynamic tumor tracking (DTT) intensity modulated radiation therapy (IMRT), with the Vero4DRT system using a moving phantom (QUASAR respiratory motion platform; QUASAR phantom) and system log files. Methods: The QUASAR phantom was placed on a treatment couch. Measurement of the point dose and dose distribution was performed for conventional IMRT, with the QUASAR phantom static and moving; for DTT IMRT, this was performed with the phantom moving for pyramid shaped, prostate, paranasal sinus, and pancreas targets. The QUASAR phantom was driven by a sinusoidal signal in the superior-inferior direction. Furthermore, predicted positional errors induced by the Vero4DRT system and mechanical positional errors of the gimbal head, were calculated using the system log files. Results and Conclusion: For DTT IMRT, the dose at the evaluation point was within $3 \%$ compared with the verification plan, and the dose distribution in the passing rates of $\gamma$ was $97.9 \%$, with the criteria of $3 \%$ dose and $3 \mathrm{~mm}$ distance to agreement. The position error calculated from the log files was within 2 $\mathrm{mm}$, suggesting the feasibility of employing DTT IMRT with high accuracy using the Vero4DRT system.
\end{abstract}

\section{Keywords}

Dynamic Tumor Tracking (DTT), Intensity Modulated Radiation Therapy (IMRT), Point Dose Verification, Dose Distribution Verification, Log File Analysis 


\section{Introduction}

Tumor respiratory motion management is essential during radiotherapy for thoracic or abdominal tumors. Expanding the area of irradiation to cover the entire volume of respiration-induced tumor motion exposes the peripheral normal tissue to high doses along with the tumor, and may lead to adverse events [1]. Methods for respiratory tumor motion management include inhalation of oxygen, abdominal compression, coaching for regular respiratory patterns, breath holding, respiratory gating, and real-time tumor-tracking [2]. Respiratory motion management is frequently associated with poor reproducibility, increased burden on the patient owing to breath holding, and/or prolonged irradiation time. Respiratory motion management of the tumor is particularly relevant to intensity modulated radiation therapy (IMRT), as it may provide excellent dose distributions for the most complex cancer volumes; it also potentially increases the therapeutic ratio drastically when the target is immobile [3]. However, the advantages of IMRT may be annulled unless organ motion is addressed; organ motion may lead to normal tissue under- or over-dosing during beam delivery, during the entire course of treatment [4].

The Vero4DRT system (Hitachi, Ltd., Tokyo, Japan) delivers treatment while the patient breathes freely, by tracking the target in real time using radiopaque fiducial markers for identification of the tumor, enabling dynamic tumor tracking (DTT). Equipped with a compact linear accelerator on the rotatory O-ring gantry and a gimbal mechanism, the light-weight X-ray head can change the beam direction [5] [6]. DTT irradiation has achieved reduced irradiation times, high irradiation reproducibility, and has reduced the burden on the patient [7]. A system of dynamic tumor tracking intensity modulated radiation therapy (DTT IMRT) has been developed by combining intensity modulated radiation therapy (IMRT) with DTT. DTT IMRT is effective for pancreatic tumors, as the radiation dose to these tumors is limited by the radiosensitivity of the surrounding gastrointestinal organs [8]; therefore, previous attempts to increase radiation doses using conventional techniques were unsuccessful, and have resulted in high morbidity and mortality [9]. However, simultaneous delivery of IMRT and DTT is expected to increase the burden on the multi-leaf collimator (MLC) and the gimbal mechanism, causing an interplay effect. Mukumoto et al. [10] verified the dose distribution during DTT IMRT using GAFCHROMIC EBT3 films (Ashland, Kentucky, USA). Depuydt, T. et al. and Garibaldi, C. et al. reported on log file analysis for DTT IMRT [11] [12]. In this study, we have verified point doses and dose distributions using an ionization chamber dosimeter and EBT3 film, respectively, during DTT IMRT. Furthermore, the precision of irradiation was calculated using the system log files.

\section{Materials and Methods}

\subsection{Dynamic Tumor Tracking Intensity Modulated Radiation Therapy Using the Vero4DRT System}

DTT was used to monitor (20 - $40 \mathrm{~s})$ the motions of infrared reflecting (IR) 
markers set on the abdominal wall and the motions of fiducial markers positioned close to the tumor at $16.7 \mathrm{~ms}$ and $320 \mathrm{~ms}$ or $640 \mathrm{~ms}$ intervals, respectively, immediately before irradiation. A four-dimensional correlation model (4D model) was then created using positions of the IR and fiducial markers [13]. The position of the fiducial markers were registered in advance on ExactracVero (Brainlab AG, Munich, Germany) using planning computed tomography (CT) images, and the positional relation to the tumor center (isocenter) was defined. During irradiation, the gimbaled X-ray head tracked the tumor based on the $4 \mathrm{D}$ model, and delivered IMRT in the step-and-shoot mode.

\subsection{Verification Plans and Irradiation Conditions}

Planning CT images were acquired using 16-slice CT BrightSpeed Elite SD (GE Healthcare UK Ltd., Buckinghamshire, England). The settings for acquisition of the planning CT were as follows: $120 \mathrm{kV}$, Auto $\mathrm{mA}$, and $0.5 \mathrm{~ms}$. The scan parameters were set as follows: $9.37 \mathrm{~mm} /$ rot helical pitch, $10 \mathrm{~mm}$ beam collimation, $16 \times 0.625 \mathrm{~mm}$ detector collimation, and $2.5 \mathrm{~mm}$ slice thickness. The IMRT plans were created using iPlan RT Dose Ver. 4.5.3 (Brainlab AG, Munich, Germany) using the X-ray voxel Monte Carlo (XVMC) algorithm for 6-MV beams. The spatial resolution and variance of dose calculation were set to $2 \mathrm{~mm}$ and $2 \%$, respectively. To investigate the effects of IMRT complexity, DTT IMRT verification plans were prepared for one case each of pyramid shaped, prostate, paranasal sinus, and pancreatic cancer targets, respectively. Details of the plans are shown in Table 1. The average mean leaf gap width [14] was calculated as an index of IMRT complexity. The average mean leaf gap width represents the segment average of the mean leaf gap width, and is calculated by dividing the gap width of each segment by the gap width of the Y-Jaw. Here, the maximum gap width of the MLC was assumed to be the gap width of the Y-Jaw, as Vero4DRT creates the irradiation field using only $5 \mathrm{~mm}$-wide MLCs. A dynamic phantom, namely, the QUASAR respiratory motion platform (Modus Medical Devices Inc., Ontario, Canada; QUASAR phantom) was then set on a treatment couch, and the I'mRT phantom (IBA Dosimetry GmbH, Schwarzenbruck, Germany) was installed on the horizontal driving shaft of the QUASAR phantom. Subsequently, a plate to which five IR markers were affixed, was set on the vertical driving shaft (Figure 1). On the upper surface of the I'mRT phantom, 4 radiopaque $\mathrm{x}$-spot (Beekley Medical, Connecticut, USA) spheres measuring $1.5 \mathrm{~mm}$ in diameter were affixed, and the positions were registered as fiducial markers on ExactracVero. The following three irradiation patterns were created using different combinations of the QUASAR phantom motion state and IMRT irradiation pattern:

a) Static IMRT: conventional IMRT irradiation with the QUASAR phantom static.

b) Non-DTT IMRT: conventional IMRT irradiation with the QUASAR phantom moving.

c) DTT IMRT: DTT IMRT irradiation with the QUASAR phantom moving. 
Table 1. Details of verification plans used in this study.

\begin{tabular}{ccccc}
\hline $\begin{array}{c}\text { IMRT plan } \\
\text { name }\end{array}$ & $\begin{array}{c}\text { Prescription } \\
\text { [Gy/fraction] }\end{array}$ & $\begin{array}{c}\text { Number } \\
\text { of ports }\end{array}$ & $\begin{array}{c}\text { Average segment } \\
\text { number per port }\end{array}$ & $\begin{array}{c}\text { PTV size } \times \text { RL } \times \text { AP } \\
{\left[\mathrm{mm}^{3}\right]}\end{array}$ \\
\hline Pyramid & 2.0 & 1 & 5.00 & none \\
Prostate & 2.0 & 7 & 14.7 & $45.0 \times 59.0 \times 41.0$ \\
Paranasal & 2.0 & 7 & 15.3 & $98.5 \times 79.0 \times 80.0$ \\
sinus & 3.0 & 5 & 14.8 & $97.5 \times 70.0 \times 42.5$ \\
Pancreas & & &
\end{tabular}

Abbreviations: PTV: planning target volume; SI: superior-inferior; RL: right-left; AP: anterior-posterior.

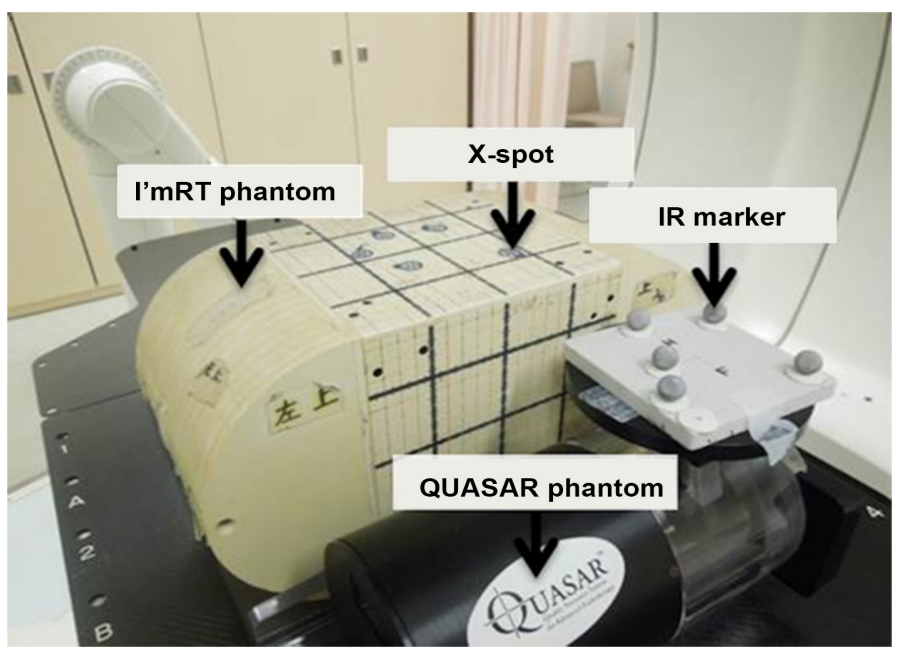

Figure 1. A photograph of the dose verification system for dynamic tumor-tracking IMRT irradiation.

In Non-DTT IMRT and DTT IMRT, the QUASAR phantom moved in the superior-inferior (SI) direction in three sinusoidal patterns [amplitude, period]: 1) $10 \mathrm{~mm}, 4 \mathrm{~s}$, 2) $20 \mathrm{~mm}, 4 \mathrm{~s}$, and 3) $40 \mathrm{~mm}, 4 \mathrm{~s}$. To verify point doses and dose distributions, and analyze the positional error of DTT IMRT, the doses were delivered at a source-axis distance of $100 \mathrm{~cm}$, and at the same gantry angle as in the actual plan for the individual irradiation patterns.

\subsection{Point Dose Verification}

An ionization chamber dosimeter CC13 (IBA Dosimetry GmbH, Schwarzenbruck, Germany) and Fluke Type 35040 (Fluke Biomedical, Ohio, USA) electrometers were employed. The active volume of the $\mathrm{CC} 13$ dosimeter is $0.13 \mathrm{~cm}^{3}$; the chamber was placed at the center of the I'mRT phantom (depth: $9 \mathrm{~cm}$ ). The measured and computed doses were then compared using the verification plan. The tolerance for dose error was set to less than $\pm 5 \%$ and $\pm 3 \%$ for single beams and summation of all beams, respectively [15].

\subsection{Dose Distribution Verification}

The dose distribution in the coronal plane was obtained by placing an EBT3 film 
at the center of the I'mRT phantom (depth: $9 \mathrm{~cm}$ ). The films were scanned in transmission mode at a resolution of $72 \mathrm{dpi}$ on the 16-bit red-channel color scale, with a constant 24-h post-exposure period using EPSON ES-10000G (SEIKO EPSON CORPORATION, Nagano, Japan). Each pixel value was normalized to the planned dose at the isocenter. The DD-system (R-TECH. INC, Nagano, Japan) was then used to analyze $\gamma$ evaluation [16]. The tolerance of the $\gamma$ pass rate, using $3 \%$ dose and $3 \mathrm{~mm}$ distance to agreement with $30 \%$ dose threshold, was set to greater than $90 \%$.

\subsection{Analysis of Positional Error of DTT IMRT Using Log Files}

The positional accuracy of DTT IMRT was estimated based on the predicted positional error of the $4 \mathrm{D}$ model, mechanical positional error of the gimbal mechanism, and baseline shifts in the patient's respiration [17]. The mean and standard deviation (SD) for the predicted and mechanical positional errors were calculated using the system log files. The position of the target recorded in the $\log$ files was assumed to be the reference position of the target on $\mathrm{kV}$ X-ray images. The difference between the reference and predicted positions of the target calculated from the positions of the IR markers and 4D model, was defined as the predicted positional error of the model [13]. Moreover, the differences between the system command and actual values at $5 \mathrm{~ms}$ intervals during DTT IMRT were defined as the mechanical positional error [7]. The correlation coefficient $\mathrm{R}$, between target velocity and acceleration for predicted and mechanical positional errors, respectively, was also determined.

\section{Results}

\subsection{Average of Mean Leaf Gap Width}

The average of mean leaf gap widths in the IMRT plan for the pyramid shaped, prostate, paranasal sinus, and pancreatic cancer targets were 38.8, 29.6, 24.5, and $26.2 \mathrm{~mm}$, respectively.

\subsection{Point Dose Verification}

The dose errors for static, Non-DTT, and DTT IMRT in the individual plans are shown in Figure 2. The dose errors for static IMRT were within the tolerance on all verification plans. For Non-DTT IMRT, the dose errors were within the tolerance only for the pyramid shaped plans. Conversely, the dose errors for the other cancer targets were within the tolerance for accumulated dose from all beams; however, the dose error from single beams exceeded the limit. In particular, larger amplitude resulted in greater standard deviations of the dose error with single beams. However, the dose errors were within the tolerance for all verification plans using DTT IMRT, and differences in dose errors by variation of amplitude were also insignificant.

\subsection{Dose Distribution Verification}

Figures 3-6 show the dose profiles for each irradiation pattern, as functions of 


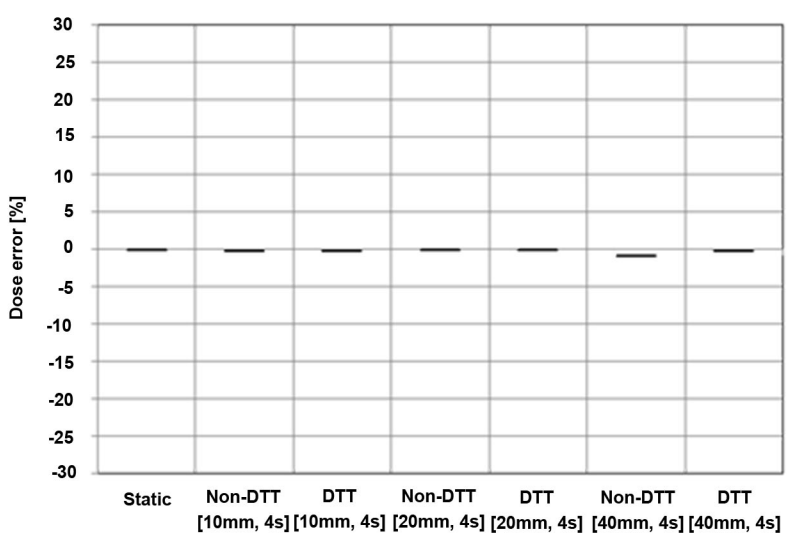

Respiratory motion [amplitude, period] (a) pyramid shaped targets

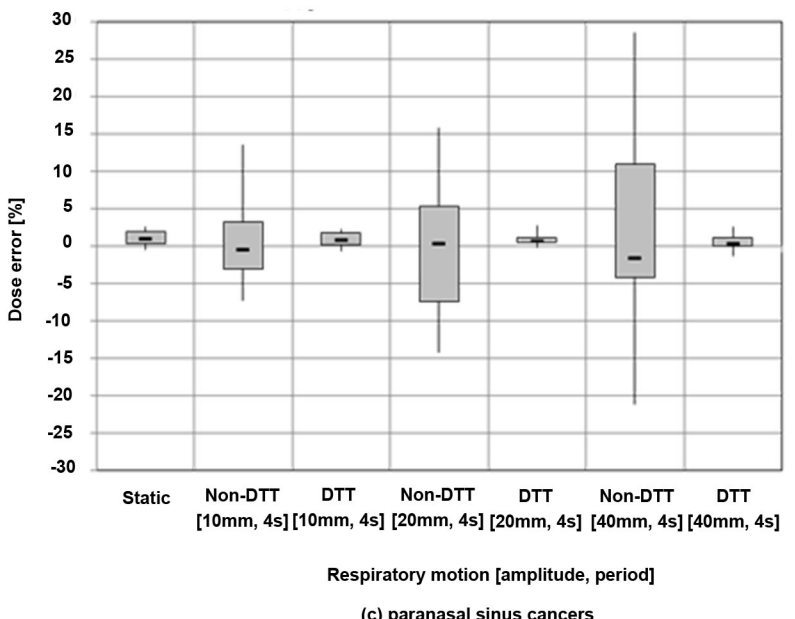

(c) paranasal sinus cancers

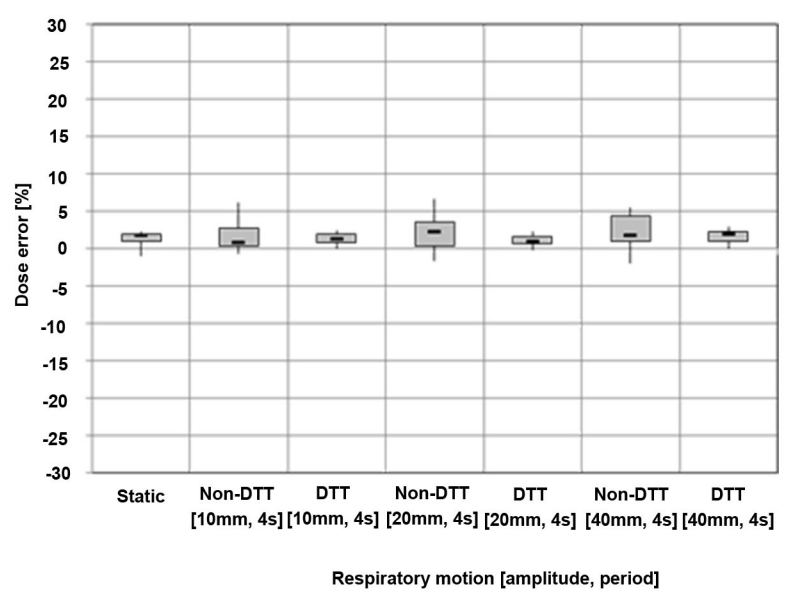

(b) prostate cancers

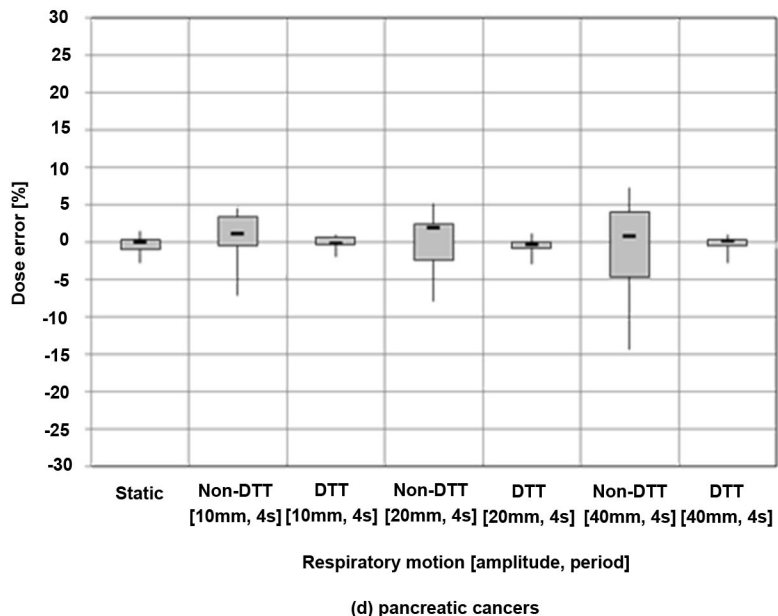

(d) pancreatic cancers

Figure 2. Result of point dose verification. The dose errors compared with verification plan among static, Non-DTT, and DTT IMRT for (a) pyramid shaped targets, (b) prostate cancers, (c) paranasal sinus cancers, (d) pancreatic cancers.

distances from the central axes. For static IMRT, the $\gamma$ pass rate was greater than $96 \%(97.9 \% \pm 1.16 \%)$ on all verification plans. For Non-DTT IMRT, compared to the verification plan, the dose profile along the superior-inferior direction, which was along the driving shaft of the QUASAR phantom, showed reductions and increases in the flat and penumbra areas, respectively, as the amplitude increased. The $\gamma$ pass rates for amplitudes of $10 \mathrm{~mm}(93.9 \% \pm 2.02 \%)$ were within the tolerance; however, they were lower than the $\gamma$ pass rates $(97.9 \% \pm 1.16 \%)$ of static IMRT. The rates at amplitudes of $20 \mathrm{~mm}$ and $40 \mathrm{~mm}$ were below the tolerance, at $68.3 \% \pm 7.82 \%$ and $45.7 \% \pm 9.50 \%$, respectively. On DTT IMRT, the dose profiles agreed well with the verification plans. The $\gamma$ pass rates were greater than $94 \%$ on all verification plans.

\subsection{Analysis of Positional Errors of DTT IMRT Using Log Files}

The predicted positional errors and velocity/acceleration of the target during DTT IMRT are shown in Table 2. Larger amplitudes led to larger predicted positional errors. The mean $+2 \mathrm{SD}$ of the predicted positional error was the largest, 

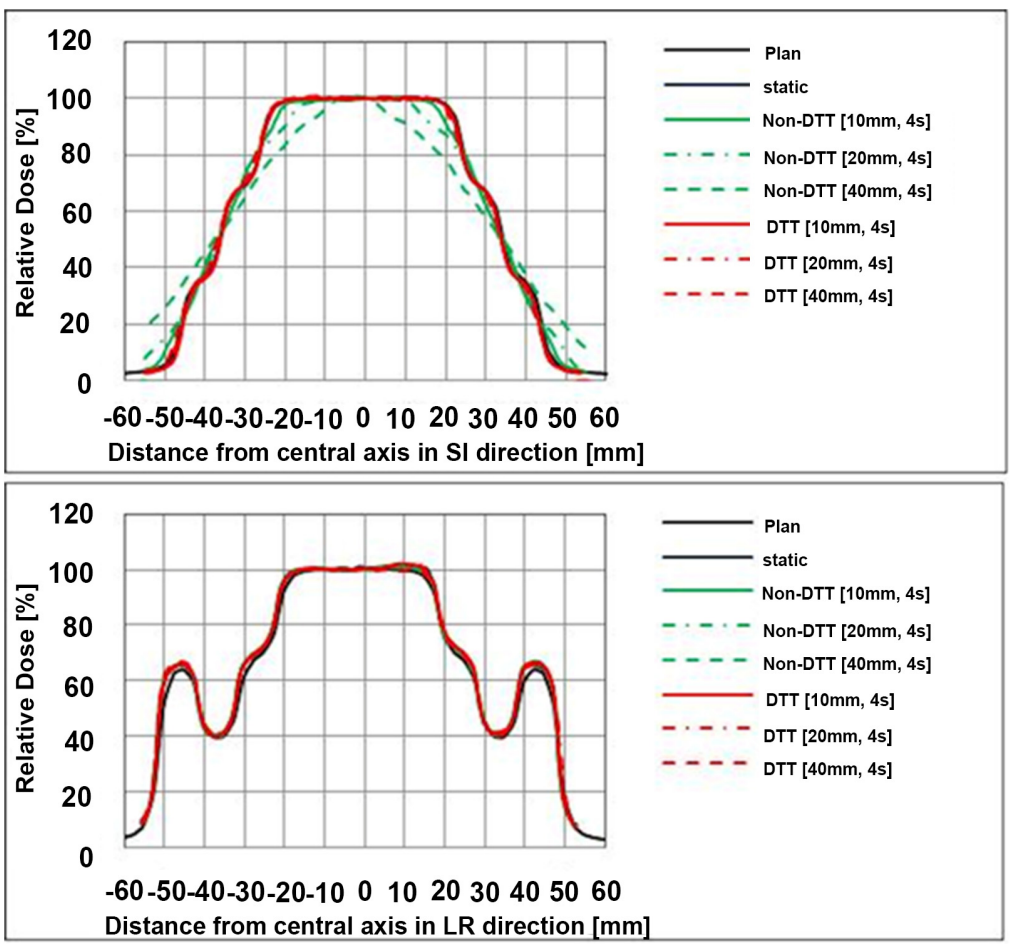

(a)

Figure 3. Result of dose distribution verification. Dose profiles as a function of distance from the central axis for pyramid shaped IMRT. (a) SI direction; (b) LR direction.
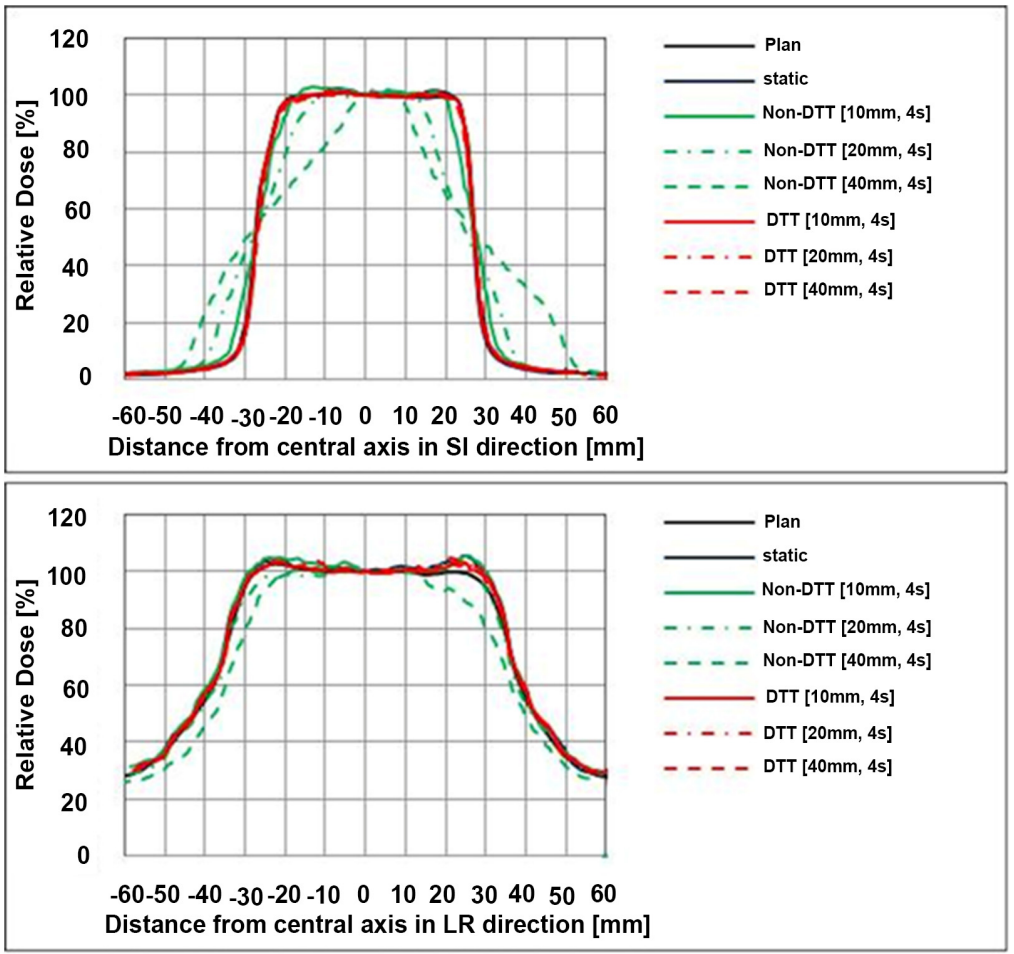

Figure 4. Result of dose distribution verification. Dose profiles as a function of distance from the central axis for prostate IMRT. (a) SI direction; (b) LR direction. 


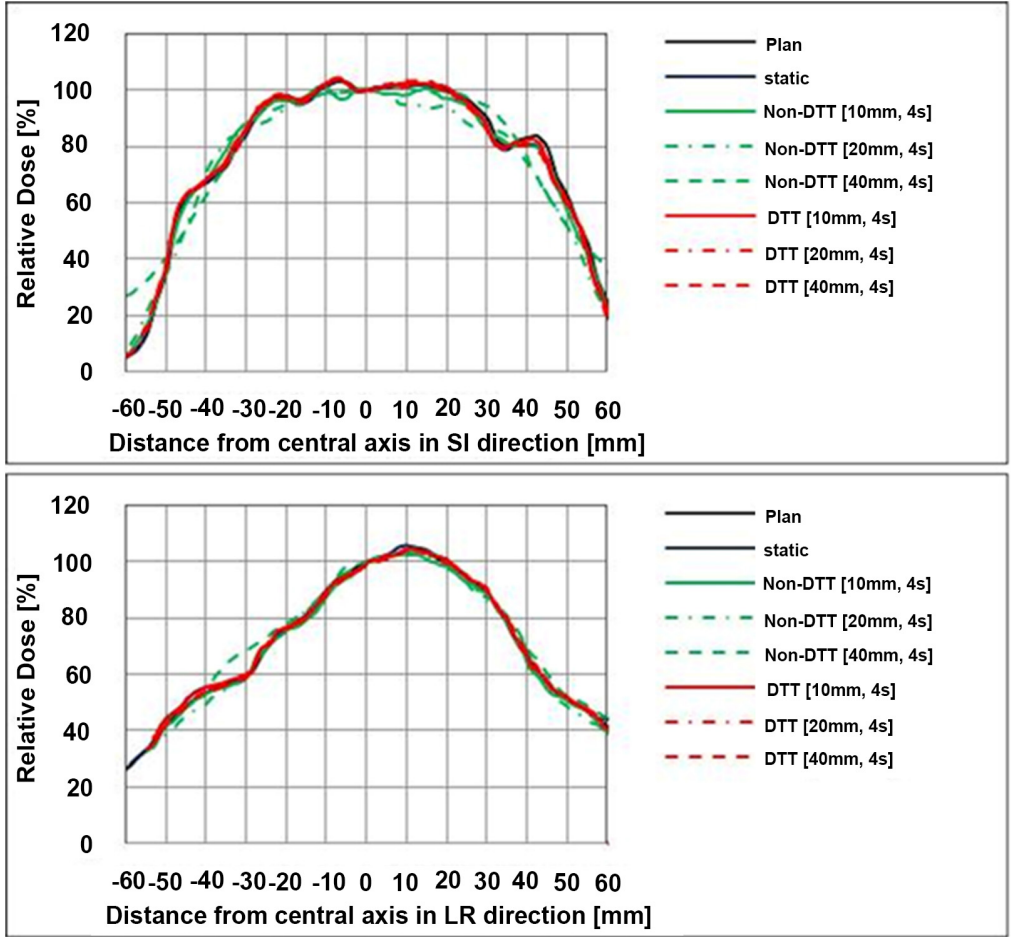

(a)

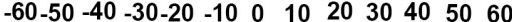

Figure 5. Result of dose distribution verification. Dose profiles as a function of distance from the central axis for paranasal sinus IMRT. (a) SI direction; (b) LR direction.
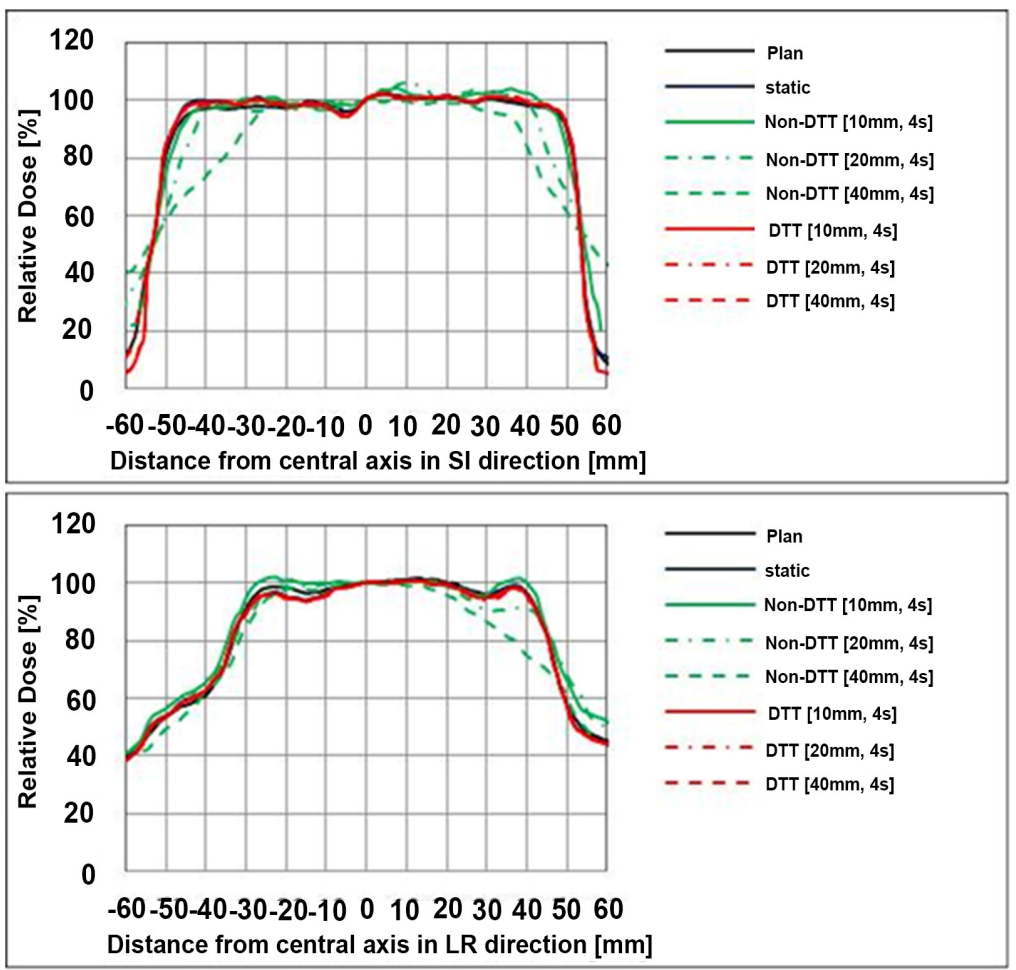

Figure 6. Result of dose distribution verification. Dose profiles as a function of distance from the central axis for pancreas IMRT. (a) SI direction; (b) LR direction. 
Table 2. Predicted positional error of the $4 \mathrm{D}$ model and correlation coefficient $\mathrm{R}$ for DTT.

\begin{tabular}{cccccc}
\hline & & \multicolumn{3}{c}{ Respiratory motion [amplitude, period] } & \\
\cline { 3 - 6 } & & {$[10 \mathrm{~mm}, 4 \mathrm{~s}]$} & {$[20 \mathrm{~mm}, 4 \mathrm{~s}]$} & {$[40 \mathrm{~mm}, 4 \mathrm{~s}]$} & $\begin{array}{c}\text { Mean } \pm \\
\text { SD }\end{array}$ \\
\hline $\begin{array}{c}\text { Predicted } \\
\text { positional error } \\
\text { of the 4D model }\end{array}$ & Mean \pm SD [mm] & $0.15 \pm 0.11$ & $0.16 \pm 0.11$ & $0.32 \pm 0.25$ & \\
& Mean + 2SD [mm] & 0.37 & 0.38 & 0.82 & \\
$\begin{array}{c}\text { Correlation } \\
\text { coefficient R }\end{array}$ & $\begin{array}{c}\text { vs velocity [mm/s] } \\
\text { vs acceleration } \\
{\left[\mathrm{mm} / \mathrm{s}^{2}\right]}\end{array}$ & 0.05 & 0.26 & 0.11 & $0.15 \pm$ \\
& & -0.36 & -0.61 & -0.75 & $\begin{array}{c}0.09 \\
0.15\end{array}$ \\
\hline
\end{tabular}

at $0.82 \mathrm{~mm}$, with an amplitude and period of $40 \mathrm{~mm}$ and $4 \mathrm{~s}$, respectively. Although the correlation coefficients between the predicted positional error and target velocity were low $(\mathrm{R}=0.15 \pm 0.01)$, those between the predicted positional error and target acceleration were high $(\mathrm{R}=-0.61 \pm 0.15)$.

The mechanical positional errors using the $4 \mathrm{D}$ model, and correlation coefficients $\mathrm{R}$ between mechanical positional errors and the velocity/acceleration of the target with DTT IMRT are shown in Table 3. The mean + 2SD of the mechanical positional error was the largest, at $1.02 \mathrm{~mm}$, with an amplitude and period of $40 \mathrm{~mm}$ and $4 \mathrm{~s}$, respectively. The correlation coefficients between mechanical positional errors and target velocities were high $(R=0.57 \pm 0.02)$, while those between mechanical positional errors and target accelerations were low $(\mathrm{R}$ $=0.36 \pm 0.02)$.

\section{Discussion}

\subsection{Point Dose Verification}

For the pyramid shaped plans, insignificant differences in dose error were observed between Non-DTT and DTT IMRT. This may be explained by the fact that the segment at the center of the pyramid had a shape that did not affect the dose even when the QUASAR phantom was driven at an amplitude of $40 \mathrm{~mm}$.

In Non-DTT IMRT, the point doses were not entirely within the tolerance limit for any of the cancer cases, even at amplitudes of $10 \mathrm{~mm}$. This was probably related to the positional difference between the MLC and target; doses were therefore delivered at different positions (phases) compared with the verification plan, resulting in an interplay effect. However, the accumulated dose from summation of all beams was within the tolerance. Mukumoto et al. also reported that the peripheral dose to the planning target volume (PTV) was reduced under moving conditions [10].

The maximum dose error per beam and SD were larger for the paranasal sinus cancers than for the other cases. This was probably related to the complexity of IMRT for paranasal sinus cancers, where the mean leaf gap was as narrow as $24.5 \mathrm{~mm}$. The smaller gap compared to the other cases was likely to cause larger dose errors. 
Table 3. Gimbal mechanical positional error and correlation coefficient R for DTT.

\begin{tabular}{|c|c|c|c|c|c|}
\hline & & \multicolumn{4}{|c|}{ Respiratory motion [amplitude, period] } \\
\hline & & {$[10 \mathrm{~mm}, 4 \mathrm{~s}]$} & {$[20 \mathrm{~mm}, 4 \mathrm{~s}]$} & {$[40 \mathrm{~mm}, 4 \mathrm{~s}]$} & $\begin{array}{c}\text { Mean } \pm \\
\text { SD }\end{array}$ \\
\hline \multirow{2}{*}{$\begin{array}{l}\text { Gimbal } \\
\text { mechanical } \\
\text { positional } \\
\text { error }\end{array}$} & Mean \pm SD $[\mathrm{mm}]$ & $0.14 \pm 0.09$ & $0.22 \pm 0.14$ & $0.44 \pm 0.29$ & \\
\hline & Mean $+2 \mathrm{SD}[\mathrm{mm}]$ & 0.32 & 0.51 & 1.02 & \\
\hline \multirow{2}{*}{$\begin{array}{l}\text { Correlation } \\
\text { coefficient R }\end{array}$} & vs velocity $[\mathrm{mm} / \mathrm{s}]$ & 0.54 & 0.58 & 0.58 & $\begin{array}{c}0.57 \pm \\
0.02\end{array}$ \\
\hline & $\begin{array}{l}\text { vs acceleration } \\
{\left[\mathrm{mm} / \mathrm{s}^{2}\right]}\end{array}$ & 0.27 & 0.35 & 0.45 & $\begin{array}{c}0.36 \pm \\
0.07\end{array}$ \\
\hline
\end{tabular}

In DTT IMRT, the point doses were consistent with that of the verification plan, and were also within the tolerance. However, the experimental system in this study was practically limited to the clinical situation on two points: 1) the motions of the target and IR markers were synchronized by the QUASAR phantom, 2) it involved motions only along the SI direction.

\subsection{Dose Distribution Verification}

Several reports [18]-[26] suggest that while irradiating during respiratory movement, the blurring effect decreases the PTV marginal dose and the penumbra expands, resulting in a difference from the planned dose distribution. The level of inconsistency depends on the respiration amplitude and complexity of intensity modulation. In this study, the profile along the SI direction, or along the driving shaft of the QUASAR phantom, showed an expanded penumbra compared to the verification plan for Non-DTT IMRT. The profile along the left-right direction was consistent with that of the pyramid shaped plan. This was probably related to the fact that the pyramid shaped plan consisted of rectangular field segments; therefore, a number of leaves had mutually similar left-right coordinates. In other plans, the fluctuations in dose distribution were directly proportional to the amplitude, and notable reductions were observed in the $\gamma$ pass rate. In DTT IMRT, the $\gamma$ pass rates were consistent with that of the verification plans, and were within the tolerance. Mukumoto et al. reported that the dosimetric accuracy showed significant improvements, of up to $92.9 \% \pm 4.0 \%$ with tracking versus $69.8 \% \pm 7.4 \%$ without tracking, using $\gamma$ pass rates with criteria of $3 \% / 1 \mathrm{~mm}(\mathrm{p}<$ 0.001). In their study, static, Non-DTT, and DTT IMRT films were compared. Conversely, in this study, the verification plans and individual films were compared. Colvill et al. conducted a multi-institutional dosimetric comparison study between real-time adaptive and non-adaptive radiotherapy [27]; respiratory motion resulted in much higher $\gamma$-fail rates without motion adaptation. However, these rates were comparable for conventional IMRT under static conditions [27] [28]. The AAPM TG-119 protocol described for IMRT commissioning showed that the action level was $88 \%-90 \%$ of the $\gamma$ pass rate, with a tolerance of $10 \%$ of the dose threshold using the $3 \% / 3 \mathrm{~mm}$ criterion [28]. According to the European 
Society for Radiotherapy and Oncology guidelines, $\gamma$ pass rates $<90 \%$ are not acceptable [29]. The dose distribution in our study was consistent with that of their study.

Doses could be delivered with accuracy equivalent to that of static IMRT; this indicates that DTT IMRT may minimize dosimetric errors from interplay, and reliable IMRT delivery may be achieved for moving targets.

\subsection{Analysis of Positional Errors of DTT IMRT Using Log Files}

The predicted positional error of the $4 \mathrm{D}$ model poorly correlated with the velocity of the target; however, it was highly correlated with target acceleration. This was probably related to the fact that increased acceleration of the target reduced the prediction accuracy of the system at the peak of the respiratory waveform (expiratory and inspiratory phases). Mukumoto et al. reported that the mean + $2 \mathrm{SD}$ of the predicted positional error of the 4D model in the Vero4DRT system was within the $2 \mathrm{~mm}$ range under conditions of synchronicity between the motion of the target and IR marker [13]. Depuydt et al. [11] reported that the tracking error $90 \%$ percentile E $(90 \%)$ was $<0.82 \mathrm{~mm}$. Garibaldi et al. [12] reported that the RMS of prediction and mechanical errors were up to $0.8,0.5$, and $0.9 \mathrm{~mm}$ for all non-phased-shifted motion patterns. Similar synchronicity was also maintained in this study, and the maximum mean $+2 \mathrm{SD}$ of the predicted positional error was $0.82 \mathrm{~mm}$, even under the severest conditions of amplitude and period of $40 \mathrm{~mm}$ and $4 \mathrm{~s}$, respectively; our results were therefore consistent with that of their study.

Mechanical positional errors were highly correlated with the velocities of the target; however, the correlation with the accelerations of the target was low. This was related to the delay in response of $5 \mathrm{~ms}$ in real time that is required by the gimbal mechanism to change the direction of the beam towards the target position, as predicted by the system during DTT irradiation. On rapid movement of the target in $5 \mathrm{~ms}$, the positional difference from the predicted position became large, resulting in high correlation between the mechanical positional error and the velocity of the target. However, even at the severest condition of amplitude and period of $40 \mathrm{~mm}$ and $4 \mathrm{~s}$, respectively, the mean $+2 \mathrm{SD}$ of the mechanical positional error was only $1.02 \mathrm{~mm}$, confirming the high accuracy of the gimbal mechanism. In practice, the system was controlled during DTT irradiation to ensure that the beam was held when the mechanical positional error exceeded 2 $\mathrm{mm}$ during delivery of megavoltage $\mathrm{X}$-rays. Also in medical practice, patients are coached to breathe slowly and smoothly (for a smooth and long respiratory waveform) to control the velocity of the target.

\section{Conclusion}

Compared to the verification plans, the point doses and dose distributions of DTT IMRT by Vero4DRT in this study were within the tolerance limits. In addition, the positional errors calculated from the log files were small, suggesting 
that DTT IMRT may deliver doses with high accuracy comparable to static IMRT.

\section{Acknowledgements}

This research was partially supported by the Japan Agency for Medical Research and Development (AMED) by grant number JP17ck0106303, JSPS KAKENHI grant number 18K07650, and Kasahara Memorial Foundation For Medical Research.

\section{Conflicts of Interest}

The authors declare no conflicts of interest regarding the publication of this paper.

\section{References}

[1] Matsuo, Y., Shibuya, K., Nakamura, M., Narabayashi, M., Sakanaka, K., Ueki, N., et al. (2012) Dose-Volume Metrics Associated with Radiation Pneumonitis after Stereotactic Body Radiation Therapy for Lung Cancer. International Journal of Radiation Oncology, Biology, Physics, 83, e545-549. https://doi.org/10.1093/jrr/rrs122

[2] Matsuo, Y., Onishi, H., Nakagawa, K., Nakamura, M., Ariji, T., Kumazaki, Y., et al. (2013) Guidelines for Respiratory Motion Management in Radiation Therapy. Journal of Radiation Research, 54, 561-568. https://doi.org/10.1093/jrr/rrs122

[3] Nakamura, A., Hiraoka, M., Itasaka, S., Nakamura, M., Akimoto, M., Ishihara, Y., et al. (2018) Evaluation of Dynamic Tumor-Tracking Intensity-Modulated Radiotherapy for Locally Advanced Pancreatic Cancer. Scientific Reports, 8, Article No. 17096. https://doi.org/10.1038/s41598-018-35402-7

[4] Keall, P.J., Mageras, G.S., Balter, J.M., Emery, R.S., Forster, K.M., Jiang, S.B., et al. (2006) The Management of Respiratory Motion in Radiation Oncology Report of AAPM Task Group 76. Medical Physics, 33, 3874-3900. https://doi.org/10.1118/1.2349696

[5] Hirata, Y., Miyamoto, N., Shimizu, M., Yoshida, M., Hiramoto, K., Ichikawa, Y., et al. (2014) International Standardization of Four Dimensional Radiotherapy System: Enhancement of Effects of Irradiation and Assurance of Safety. Synthesiology, 7, 238-246. https://doi.org/10.5571/synth.7.238

[6] Kamino, Y., Takayama, K., Kokubo, M., Narita, Y., Hirai, E., Kawawda, N., et al. (2006) Development of a Four-Dimensional Image-Guided Radiotherapy System with a Gimbaled X-Ray Head. International Journal of Radiation Oncology, Biology, Physics, 66, 271-278. https://doi.org/10.1016/j.ijrobp.2006.04.044

[7] Mukumoto, N., Nakamura, M., Sawada, A., Takahashi, K., Miyabe, Y., Takayama, K., et al. (2012) Positional Accuracy of Novel X-Ray Image-Based Dynamic Tumor-Tracking Irradiation Using a Gimbaled MV X-Ray Head of a Vero4DRT (MHI-TM2000). Medical Physics, 39, 6287-6296. https://doi.org/10.1118/1.4754592

[8] Goto, Y., Ashida, R., Nakamura, A., Itasaka, S., Shibuya, K., Akimoto, M., et al. (2018) Clinical Results of Dynamic Tumor Tracking Intensity-Modulated Radiotherapy with Real-Time Monitoring for Pancreatic Cancers Using a Gimbal Mounted Linac. Oncotarget, 9, 23628-23635. https://doi.org/10.18632/oncotarget.25310

[9] Chauffert, B., Mornex, F., Bonnetain, F., Rougier, P., Mariette, C., Bouche, O., et al. 
(2008) Phase III Trial Comparing Intensive Induction Chemoradiotherapy (60 Gy, Infusional 5-FU and Intermittent Cisplatin) Followed by Maintenance Gemcitabine with Gemcitabine Alone for Locally Advanced Unresectable Pancreatic Cancer. Definitive Results of the 2000-01 FFCD/SFRO Study. Annals of Oncology, 19, 1592-1599. https://doi.org/10.1093/annonc/mdn281

[10] Mukumoto, N., Nakamura, M., Yamada, M., Takahashi, K., Akimoto, M., Miyabe, Y., et al. (2016) Development of a Four-Axis Moving Phantom for Patient-Specific QA of Surrogate Signal-Based Tracking IMRT. Medical Physics, 43, 6364. https://doi.org/10.1118/1.4966130

[11] Depuydt, T., Verellen, D., Haas, O., Gevaert, T., Linthout, N., Duchateau, M., et al. (2011) Geometric Accuracy of a Novel Gimbals Based Radiation Therapy Tumor Tracking System. Radiotherapy and Oncology, 98, 365-372.

https://doi.org/10.1016/j.radonc.2011.01.015

[12] Garibaldi, C., Russo, S., Ciardo, D., Comi, S., Seregni, M., Fassi, A., et al. (2015) Geometric and Dosimetric Accuracy and Imaging Dose of the Real-Time Tumour Tracking System of a Gimbal Mounted Linac. Physica Medica, 31, 501-509. https://doi.org/10.1016/j.ejmp.2015.04.001

[13] Mukumoto, N., Nakamura, M., Sawada, A., Suzuki, Y., Takahashi, K., Miyabe, Y., et al. (2013) Accuracy Verification of Infrared Marker-Based Dynamic Tumor-Tracking Irradiation Using the Gimbaled X-Ray Head of the Vero4DRT (MHI-TM2000). Medical Physics, 40, Article ID: 041706. https://doi.org/10.1118/1.4794506

[14] Tatsumi, D., Hosono, M.N., Nakada, R., Ishii, K., Tsutsumi, S., Inoue, M., et al. (2011) Direct Impact Analysis of Multi-Leaf Collimator Leaf Position Errors on Dose Distributions in Volumetric Modulated Arc Therapy: A Pass Rate Calculation between Measured Planar Doses with and without the Position Errors. Physics in Medicine and Biology, 56, N237-N246. https://doi.org/10.1088/0031-9155/56/20/N03

[15] Japan Society for Radiation Oncology Quality Assurance Committee (2004) Guideline about Securing the Mechanical Precision of Intensity-Modulated Radiation Therapy Using Multi-Leaf Collimator (Ver. 1). The Official Journal of the Japanese Society for Therapeutic Radiology and Oncology, 16, 197-203.

[16] Low, D.A., Harms, W.B., Mutic, S. and Purdy, J.A. (1998) A Technique for the Quantitative Evaluation of Dose Distributions. Medical Physics, 25, 656-661. https://doi.org/10.1118/1.598248

[17] Kito, S. (2014) Actual Practices of Dynamic Tumor Tracking Irradiation at Komagome Hospital. Journal of Radiotherapy Section of Japanese Society of Radiological Technology, 28, 43-48. (In Japanese)

[18] Li, X., Yang, Y., Li, T., Fallon, K., Heron, D.E. and Huq, M.S. (2013) Dosimetric Effect of Respiratory Motion on Volumetric-Modulated Arc Therapy-Based Lung SBRT Treatment Delivered by TrueBeam Machine with Flattening Filter-Free Beam. Journal of Applied Clinical Medical Physics, 14, 4370. https://doi.org/10.1120/jacmp.v14i6.4370

[19] Chui, C.S., Yorke, E. and Hong, L. (2003) The Effects of Intra-Fraction Organ Motion on the Delivery of Intensity-Modulated Field with a Multileaf Collimator. Medical Physics, 30, 1736-1746. https://doi.org/10.1118/1.1578771

[20] Gierga, D.P., Chen, G.T., Kung, J.H., Betke, M., Lombardi, J. and Willett, C.G. (2004) Quantification of Respiration-Induced Abdominal Tumor Motion and Its Impact on IMRT Dose Distributions. International Journal of Radiation Oncology, Biology, Physics, 58, 1584-1595. https://doi.org/10.1016/j.ijrobp.2003.09.077 
[21] Bortfeld, T., Jokivarsi, K., Goitein, M., Kung, J. and Jiang, S.B. (2002) Effects of Intra-Fraction Motion on IMRT Dose Delivery: Statistical Analysis and Simulation. Physics in Medicine and Biology, 47, 2203-2220. https://doi.org/10.1088/0031-9155/47/13/302

[22] Yang, J.N., Mackie, T.R., Reckwerdt, P., Deasy, J.O. and Thomadsen, B.R. (1997) An Investigation of Tomotherapy Beam Delivery. Medical Physics, 24, 425-436. https://doi.org/10.1118/1.597909

[23] Jiang, S.B., Pope, C., Al Jarrah, K.M., Kung, J.H., Bortfeld, T. and Chen, G.T. (2003) An Experimental Investigation on Intra-Fractional Organ Motion Effects in Lung IMRT Treatments. Physics in Medicine and Biology, 48, 1773-1784. https://doi.org/10.1088/0031-9155/48/12/307

[24] Seco, J., Sharp, G.C., Turcotte, J., Gierga, D., Bortfeld, T. and Paganetti, H. (2007) Effects of Organ Motion on IMRT Treatments with Segments of Few Monitor Units. Medical Physics, 34, 923-934. https://doi.org/10.1118/1.2436972

[25] Ehler, E.D., Nelms, B.E. and Tome, W.A. (2007) On the Dose to a Moving Target While Employing Different IMRT Delivery Mechanisms. Radiotherapy and Oncology, 83, 49-56. https://doi.org/10.1016/j.radonc.2007.02.007

[26] Kang, H., Yorke, E.D., Yang, J., Chui, C.S., Rosenzweig, K.E. and Amols, H.I. (2010) Evaluation of Tumor Motion Effects on Dose Distribution for Hypofractionated Intensity-Modulated Radiotherapy of Non-Small-Cell Lung Cancer. Journal of Applied Clinical Medical Physics, 11, 3182. https://doi.org/10.1120/jacmp.v11i3.3182

[27] Colvill, E., Booth, J., Nill, S., Fast, M., Bedford, J., Oelfke, U., et al. (2016) A Dosimetric Comparison of Real-Time Adaptive and Non-Adaptive Radiotherapy: A Multi-Institutional Study Encompassing Robotic, Gimbaled, Multileaf Collimator and Couch Tracking. Radiotherapy and Oncology, 119, 159-165. https://doi.org/10.1016/j.radonc.2016.03.006

[28] Ezzell, G.A., Burmeister, J.W., Dogan, N., LoSasso, T.J., Mechalakos, J.G., Mihailidis, D., et al. (2009) IMRT Commissioning: Multiple Institution Planning and Dosimetry Comparisons, a Report from AAPM Task Group 119. Medical Physics, 36, 5359-5373. https://doi.org/10.1118/1.3238104

[29] Alber, M., Broggi, S., Wagter, C.D., Eichwurzel, I., Engström, P., Fiorino, C., et al. (2008) Guidelines for the Verifi Cation of IMRT. ESTRO Booklet No. 9. 